\title{
Self-Interference Cancellation-Based Mutual-Coupling Model for Full-Duplex Single-Channel MIMO Systems
}

\author{
Pawinee Meerasri, Peerapong Uthansakul, and Monthippa Uthansakul \\ School of Telecommunication Engineering, Suranaree University of Technology, Muang, Nakhon Ratchasima 30000, Thailand \\ Correspondence should be addressed to Peerapong Uthansakul; uthansakul@sut.ac.th
}

Received 26 July 2013; Revised 1 December 2013; Accepted 2 December 2013; Published 16 January 2014

Academic Editor: Hon Tat Hui

Copyright (C) 2014 Pawinee Meerasri et al. This is an open access article distributed under the Creative Commons Attribution License, which permits unrestricted use, distribution, and reproduction in any medium, provided the original work is properly cited.

\begin{abstract}
The challenge of a full-duplex single-channel system is the method to transmit and receive signals simultaneously at the same time and on the same frequency. Consequently, a critical issue involved in such an operation is the resulting self-interference. Moreover, for MIMO system, the full-duplex single-channel system is subjected to the very strong self-interference signals due to multiple transmitting and receiving antennas. So far in the pieces of literature, there have not been any suitable techniques presented to reduce the self-interference for full-duplex single-channel MIMO systems. This paper initially proposes the method to cancel the self-interference by utilizing the mutual-coupling model for self-interference cancellation. The interference can be eliminated by using a preknown interference, that is, the mutual-coupling signals. The results indicate that the channel capacity performance of the proposed technique can significantly be improved due to the reduction of the self-interference power. The measurement results indicate that the proposed MIMO system can suppress the self-interference and mutual-interference signals with the reduction of $31 \mathrm{~dB}$ received power.
\end{abstract}

\section{Introduction}

Nowadays, multiple-input multiple-output (MIMO) system is the promising technology for the next generation of wireless communication systems as MIMO system can provide a wide coverage area, a high spectral efficiency, and an increased system capacity. The MIMO system employs the multiple antennas to transmit signals on the same frequency which cause the strong interference signals at the receiving antennas on the same side. These interferences are more pronounced when operating the full-duplex single-channel MIMO system.

The full-duplex single-channel system is one of the most interesting technologies for future wireless communications because it can offer double throughput from any conventional system without paying any expenses of spectrum. This is because the system is able to receive and transmit simultaneously within a single channel. In the literature, the problem of full-duplex interference has been addressed on the specific configuration of MIMO relay nodes. The self-interference cancellation is introduced to be used at only relaying node
[1-3]. So far there have not been any techniques proposed for source or destination. In this light, the authors propose the new technique to suppress the self-interference for fullduplex single-channel MIMO systems. From the literature on RF interference cancellation, the work in [4-9] presents a fullduplex wireless system that can transmit and receive signals at the same time and on the same frequency band since it requires at least two antennas having one for transmitter ( $\mathrm{Tx}$ ) and one for receiver $(\mathrm{Rx})$. The key challenge in realizing such a system lies in addressing the self-interference generated by the Tx antenna at the $\mathrm{Rx}$ antenna. For example, one can implement the above self-interference cancellation idea completely in analog domain using noise cancellation circuits reported by Radunovic et al. [5]. But the practical noise cancellation circuits can only handle a dynamic range of at most $\sim 30 \mathrm{~dB}$. Another technique in [6] employs the antenna cancellation by using three antennas to create a beam forming null. This method cancels the self-interference at the receiver antenna by using antenna placement as an additional cancellation technique or antenna cancellation. The antenna cancellation requires two asymmetrically placed transmitting 
antennas and one receiving antenna. This three-antenna system can remove $\sim 60 \mathrm{~dB}$ reduction of self-interference power for a802.15.4 system. Although it looks promising, the antenna cancellation-based designs have two major limitations. The first is that they require three antennas having two transmitting antennas and one receiving antenna, which are very sensitive to the relative location of antennas and any material around them. It is a fact that the full-duplex system can have double throughput, but with three antennas a MIMO system can have triple throughput. Hence, the use of multiple antennas for only full-duplex purpose is not worth. The second limitation is a bandwidth constraint, a theoretical limit which prevents supporting wideband signal such as WiFi.

The MIMO techniques for wireless communications have been studied extensively over the past decade as a means of achieving significant capacity gains needed for supporting high-rate wireless broadband applications [10]. A critical factor in the design and analysis of MIMO systems is the theoretical models which are used for representing the MIMO transceiver as well as the wireless fading channel. So far in the literature, the factor on realistic channel configuration has gained a lot of attention such as spatial correlation (see, e.g., $[11,12]$ and many others). One issue which has received less attention in comparison is that of mutual coupling [10, 13-15], which occurs due to electromagnetic interactions between the antennas in both transmitter and receiver. This effect, as well as spatial correlation, is particularly significant for applications with compact antennas, such as cellular mobile, in which the available space for placing the antennas is highly restrictive.

In this paper, we will investigate the effect of antenna mutual coupling (MC) on the full-duplex single-channel MIMO system with the aim of self-interference eliminations. Based on the mutual-coupling model, the signals with selfinterference can be preknown. As a result, it is possible to eliminate all self-interference signals by subtracting from preknown signals. The concept of transmitting and receiving mutual impedances is employed to incorporate the antenna MC effect into the correlated channel model [16]. This model is applied to work out the suppression technique to reduce the self-interference performed by subtracting the interference signals from the transmitting signals that are suitably tuned according to the interaction between multiple antennas. This is because the self-interference signals do not depend on the environments. Then, the proposed technique can be done on the manufacturing process. The paper presents the comparison between the MC full-duplex single-channel systems with and without the proposed technique. The channel capacity performance is the key performance used to indicate the merit of proposed technique. The results show that the proposed technique is not only to suppress the interference but also to improve the system performance capacity.

\section{Problem Formulation}

This paper focuses on the full-duplex wireless communications operating on the same frequency and at the same time.
The simultaneous transmitting and receiving signals can be achieved via the cancellation of the self-interference signal. However, the problem is that the self-interference is billions of times stronger (60-90 dB) than a received signal; for example, for WiFi the self-interference would be nearly up to $80 \mathrm{~dB}$ stronger. Hence, the main key success is to eliminate the selfinterference as much as possible. In this section, the overview of full-duplex system is presented in order to be the basic knowledge before getting to the main problem of this work. Next, the survey of RF interference cancellation techniques is detailed.

2.1. Full-Duplex Wireless Communication. Currently, fullduplex wireless systems achieve the isolation required between the two directions of communication using independence in either time or frequency. Accordingly, these duplexings are called time division duplexing (TDD) and frequency division duplexing (FDD). The TDD system is the system that divides the access of each node in time. TDD is also commonly known as half-duplexing. Other fullduplex wireless systems separate the Tx and $\mathrm{Rx}$ functions in the frequency domain, the so-called FDD, and may operate using two different carrier frequencies for carrying transmissions. In this case, nodes 1 and 2 can send data to each other at the same time, although using two different frequencies. The use of different frequencies prevents the two signals from interfering with each other, even though the two transmissions occur at the same time. Time division duplexing exacerbates the inconsistency in the channel views across nodes. Since only one node among a pair of communicating nodes can transmit at a given time, the wireless channel around the transmitting node may look occupied, while the wireless channel around the receiving node may look unoccupied. Such inconsistencies are the root cause of many of the problems with time division duplexing wireless networks, such as packet losses due to hidden terminal effects. On the other hand, frequency division duplexing requires a wireless node to use twice the frequency bandwidth for sending and receiving signals of a given bandwidth. In some cases, this is expensive and infeasible. The key challenge in implementing a full-duplex wireless system, where a device can simultaneously transmit and receive signals over-the-air at the same time and in the same frequency band, is the large power differential between the self-interference from a node's own transmission and the signal of interest coming from a distant source.

2.2. Single-Channel Full-Duplex Wireless Communications. A basic perception of wireless communication is that a radio cannot transmit and receive on the same frequency and at the same time. As wireless signals attenuate quickly over distance, the signal from a local transmitting antenna is hundreds of thousands of times stronger than transmissions from other nodes. Figure 1 shows an example where nodes 1 and 2 are trying to send data to each other simultaneously using the same frequency. Node 1 own transmission is much stronger at its receiving antenna, compared to the signal it receives from node 2 . With such strong self-interference, the receiver 


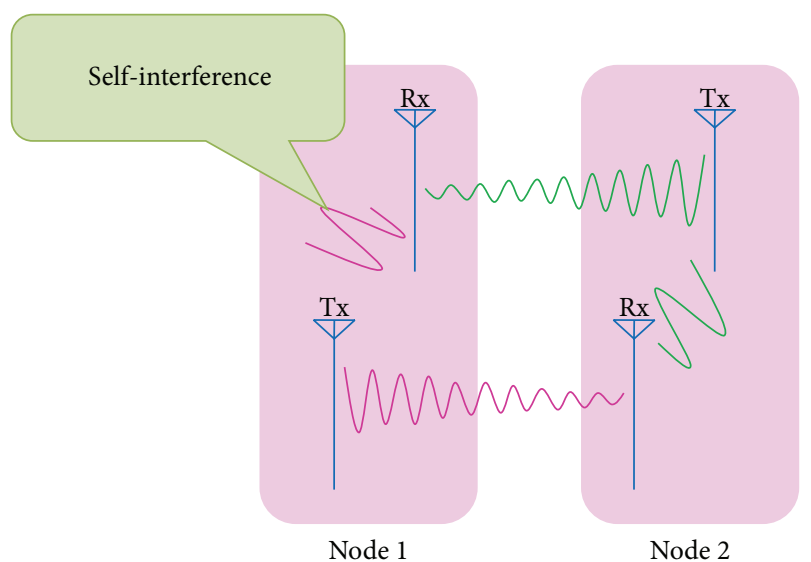

FIGURE 1: Self-interference in the single-channel full-duplex wireless communications using one transmitting antenna and one receiving antenna.

of node 1 is unable to decode any signals that node 2 is trying to send to node 1 . This example shows that the biggest challenge in designing single-channel full-duplex wireless communications is to eliminate the self-interference signal from the receiver of the wireless node. In theory, this problem should be easy to solve. For a system with antennas each for transmitting and receiving, since the system knows the signal of transmitting antenna, it can subtract this from the signal of receiving antenna and decode the remainder.

2.3. Self-Interference Cancellation. The work in [17] proposed the design of full-duplex system that requires only one antenna using circulator to share the same antenna for transmitting and receiving paths as shown in Figure 2. The selfinterference cancellation (SIC) uses the knowledge of transmission to cancel self-interference in the RF signal before it is digitized. In an ideal analog cancellation scenario, the amplitudes from the two paths would be perfectly matched at the receiver and phase of the two signals would differ by exact $\pi$. To cancel self-interference, the best performing prior design is obtained. The authors gain the inverse of the transmitted signal using a phase shifter with attenuator. The attenuator and phase shifter allow a modulator to control the angle and amplitude of a feed signal.

\section{System Model}

3.1. MIMO Model. In this section, the capacity formula of MIMO systems is briefly given. We assume an independent and identically distributed (i.i.d.) Rayleigh flat-fading channel in rich scattering environments, and the channel is unknown at the transmitter and perfectly known at the receiver. The basic MIMO structure is depicted in Figure 3. Let the number of transmitting and receiving antennas be $N_{T}$ and $M_{R}$, respectively. We denote this MIMO communication link as $\left(N_{T}, M_{R}\right)$. The $M_{R} \times 1$ received signal vector $\mathbf{y}$ can be written as

$$
\mathbf{y}=\mathbf{H x}+\mathbf{n},
$$

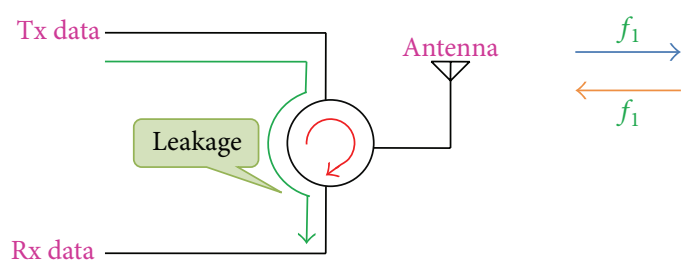

FIGURE 2: Self-interference in the single-channel full-duplex singleantenna wireless communications.

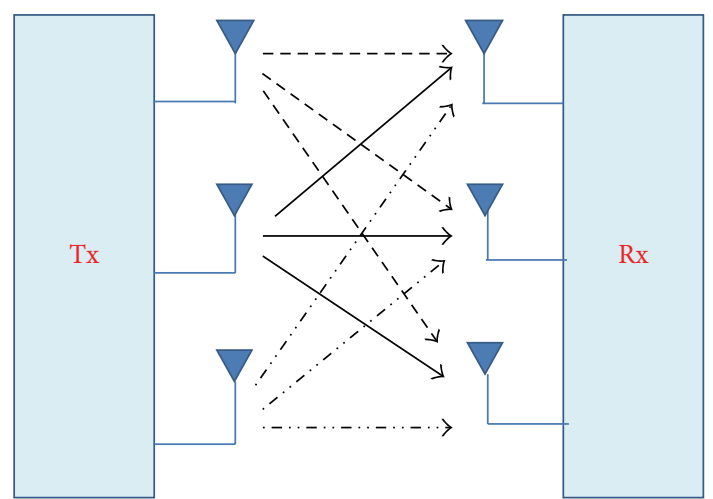

FIGURE 3: Basic structure of MIMO system.

with this notation channel output sequence that can be written in matrix form as

$$
\left[\begin{array}{c}
y_{1} \\
y_{2} \\
\vdots \\
y_{M_{R}}
\end{array}\right]=\left[\begin{array}{cccc}
h_{11} & h_{12} & & h_{1 N_{T}} \\
h_{21} & h_{22} & \cdots & h_{2 N_{T}} \\
\vdots & \vdots & \ddots & \vdots \\
h_{M_{R^{1}}} & h_{M_{R^{2}}} & \cdots & h_{M_{R^{N} T}}
\end{array}\right]\left[\begin{array}{c}
x_{1} \\
x_{2} \\
\vdots \\
x_{N_{T}}
\end{array}\right]+\left[\begin{array}{c}
n_{1} \\
n_{2} \\
\vdots \\
n_{M_{R}}
\end{array}\right] \text {, }
$$

where $\mathbf{H}$ is $M_{R} \times N_{T}$ channel matrix with the entry $h_{i, j}$ describing the channel gain between the $j$ th transmitting antenna and the $i$ th receiving antenna, $\mathbf{x}$ is $N_{T} \times 1$ transmitted signal vector with independent symbols, and $\mathbf{n}$ is $M_{R} \times 1$ additive white Gaussian noise (AWGN) vector.

The AWGN vector $\mathbf{n}$ satisfies $E\left\{\mathbf{n} \mathbf{n}^{H}\right\}=\mathbf{I}_{M_{R}}$ in which $\mathbf{n}^{H}$ denotes the conjugate transpose of $\mathbf{n}$ and $\mathbf{I}_{M_{R}}$ denotes $M_{R} \times$ $M_{R}$ identity matrix.

As the channel is unknown at the transmitter, equal power is allocated to each of the transmitting antennas. Then the MIMO capacity in bits per second per Hertz $(\mathrm{bps} / \mathrm{Hz})$ is derived as

$$
C=\log _{2} \operatorname{det}\left(\mathbf{I}_{M_{R}}+\frac{\rho}{N_{T}} \mathbf{H H}^{H}\right),
$$

where $\rho$ is the average received signal to noise ratio (SNR). $\mathbf{H}$ is normalized channel matrix [18].

3.2. Mutual-Coupling Effects on MIMO. In this section, in order to support parallel signal transmission in a MIMO system, the antennas at transmitter and receiver have to be properly coupled to the modes offered by the wireless communication channel. Hence, in Figure 4, the array elements 


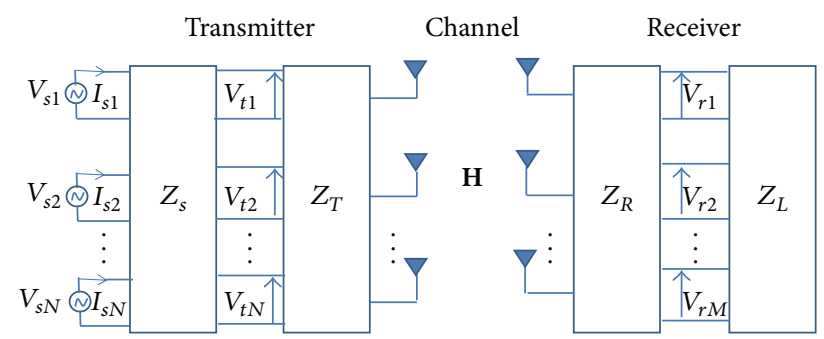

Figure 4: An $M_{R} \times N_{T}$ MIMO system based on mutual-coupling model.

location (including spacing and orientation) with respect to the scatterers is of paramount importance in the operation of the MIMO system. The interactions between the entire set of antennas and scatterers are initially described by the impedance matrix $\mathbf{Z}$. For dipoles, however, the mutual impedance can easily be calculated using classical induced electromagnetic force (EMF) method [19]. The value of the mutual impedance between the $m$ th and $n$th dipoles $Z_{m n}$ is given by [20]

$$
Z_{m n}=\left\{\begin{array}{cc}
30\left[0.5772+\ln \left(\beta d_{\mathrm{lam}}\right)-C_{i}\left(\beta d_{\mathrm{lam}}\right)\right] & \\
+j\left[30 S_{i}\left(\beta d_{\mathrm{lam}}\right)\right] & m=n, \\
30\left[2 C_{i}\left(u_{0}\right)-C_{i}\left(u_{1}\right)-C_{i}\left(u_{2}\right)\right] & \\
-j\left[30\left(2 S_{i}\left(u_{0}\right)-S_{i}\left(u_{1}\right)-S_{i}\left(u_{2}\right)\right)\right] & m \neq n,
\end{array}\right.
$$

where $\beta=2 \pi / d_{\text {lam }}$ is the wave number, $d_{\text {lam }} / 2$ is the dipole length, and the constants are given by [20]

$$
\begin{gathered}
u_{0}=\beta d_{h}, \\
u_{1}=\beta\left(\sqrt{d_{h}^{2}+\left(d_{\mathrm{lam}} / 2\right)^{2}}+\left(d_{\mathrm{lam}} / 2\right)\right), \\
u_{2}=\beta\left(\sqrt{d_{h}^{2}+\left(d_{\mathrm{lam}} / 2\right)^{2}}-\left(d_{\mathrm{lam}} / 2\right)\right),
\end{gathered}
$$

where $d_{h}$ is the horizontal distance between the two dipole antennas and $C_{i}(u)$ and $S_{i}(u)$ are the cosine and sine integrals, respectively:

$$
C_{i}(u)=\int_{\infty}^{u} \frac{\cos (x)}{x} d x, \quad S_{i}(u)=\int_{0}^{u} \frac{\sin (x)}{x} d x .
$$

It has to be noted that, while calculating $Z_{m n}$, we assume that the $n$th dipole is excited with current, while all the remaining dipoles are open circuited.

In general, mutual coupling can be characterized by numerical modelling techniques [19]. However, for dipoles, we can use analytical mutual coupling into the MIMO system model. The coupling matrix of transmitting antenna array $\mathbf{C}_{T}$ can be written using fundamental electromagnetic and circuit theory [19]. $\mathbf{C}_{T}$ has the meaning of transfer function matrix for the transmitting array and is given as

$$
\mathbf{C}_{T}=\left(Z_{A}+Z_{T}\right)\left(\mathbf{Z}+Z_{T} \mathbf{I}_{N_{T}}\right)^{-1}
$$

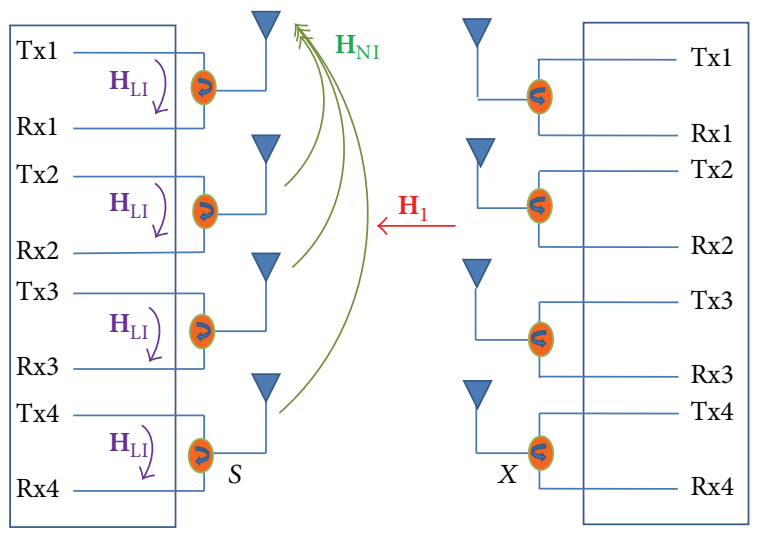

FIGURE 5: Model of full-duplex single-channel $4 \times 4$ MIMO system.

where $Z_{A}$ is the element's impedance in isolation. The element $Z_{m n}$ of matrix $\mathbf{Z}$ is defined by using the EMF method as described in (4). Also the coupling matrix of receiving antenna array $\mathbf{C}_{R}$ can be determined in a similar manner. $\mathbf{C}_{R}$ has the meaning of transfer function matrix for the receiving array and is given as

$$
\mathbf{C}_{R}=\left(Z_{A}+Z_{T}\right)\left(\mathbf{Z}+Z_{T} \mathbf{I}_{M_{R}}\right)^{-1} .
$$

\section{Proposed Self-Interference Cancellation}

In this section, we consider a generic MIMO radio unit equipped with $M_{R}$ RF receivers antennas and $N_{T}$ RF signal generators/transmitters. Among all generators, there are $N_{s}=$ $N_{T}-M_{R}$ primary generators and $M_{R}$ auxiliary generators. The primary generators are used to transmit up to $N_{S}$ independent streams of data. The auxiliary generators are used to generate RF waveforms for SIC at the RF frontend of the receivers on the same frequency. See Figure 5.

Furthermore, we index the receiver by $k=1, \ldots, M_{R}$ and the transmitter by $k=M_{R}+1, \ldots, N_{T}$. Then, for each transmitted data packet subject to linear modulation, a RF signal stream transmitted from the $k$ th generator ideally can be expressed by $\widetilde{x_{k}}(t)=\operatorname{Re}\left\{x_{k}(t) \exp \left(j 2 \pi f_{c} t\right)\right\}$, where $f_{c}$ is the carrier frequency and

$$
x_{k}(t)=\sum_{i=1}^{I} g_{k}^{(i)}(t) * \sum_{n=-L}^{N-1} s_{n}^{(i)} p(t-n T)
$$

where $x_{k}(t)$ is the complex baseband form (also called $I / Q$ waveform) of $\widetilde{x_{k}}(t)$. Here, $g_{k}^{(i)}(t)$ is the complex impulse response of the $k$ th transmission for data stream $i$ (of total $I$ streams), $s_{n}^{(i)}$ is the complex symbol sequence for data stream $i, N+L$ is the number of complex symbols per stream (including the $L$ prefixed symbols as used in OFDM system), and $p(t)$ is the fundamental pulse waveform used for linear pulse modulation, which has the double-sided bandwidth $W$ and the effective duration $T$. For high spectral efficiency, it is typical that $T$ is equal to or only slightly larger than $1 / W$. The operator $*$ denotes convolution. 


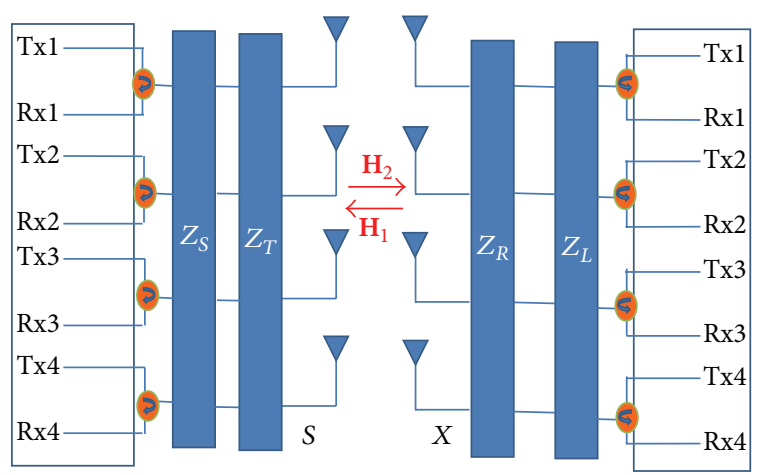

Figure 6: $4 \times 4$ MIMO system with mutual-coupling model.

The RF self-interference received by the by the $l$ th receiver is $\widetilde{y}_{l}(t)=\operatorname{Re}\left\{y_{l}(t) \exp \left(j 2 \pi f_{c} t\right)\right\}$, where $l=1, \ldots, M_{R}$, and $y_{l}(t)=\sum_{k=1}^{N_{T}} h_{\mathrm{mc}_{l, k}}(t) * x_{k}(t)=\sum_{i=1}^{I}\left(\left[\sum_{k=1}^{N_{T}} h_{\mathrm{mc}_{l, k}}(t) * g_{k}^{(i)}(t)\right] *\right.$ $\left.\sum_{n=0}^{N-1} s_{n}^{(i)} t(t-n T)\right)$ is the I/Q waveform of $\widetilde{y}_{l}(t)$. In Figure 6, when the mutual coupling is presented, $h_{l, k}(t)$ is the complex baseband channel impulse response from the $k$ th generator to the $l$ th receiver on the same radio. Hence, the channel matrix $h_{l, k}(t)$ obtained from the case that this effect is absent has to be pre- and postmultiplied by coupling matrices $\mathbf{C}_{R}$ and $\mathbf{C}_{T}$. As a result, the new channel matrix is given by $\mathbf{H}_{\mathrm{mc}}=\mathrm{C}_{R} \mathbf{H C}_{T}$. To cancel the RF self-interference $\widetilde{y}_{l}(t)$ for all $l$ and $t$, it is necessary to find $g_{k}^{(i)}(t)$ for all $k$ and $i$ such that $y_{l}(t)=0$ for all $l$ or equivalently $\sum_{k=1}^{N_{T}} h_{\mathrm{mc}_{l, k}}(t) * g_{k}^{(i)}(t)=0$ for all $l$ and $i$. The matrix form of this condition is

$$
\left[\begin{array}{ccc}
h_{\mathrm{mc}_{1,1}}(t) & \cdots & h_{\mathrm{mc}_{1, N_{T}}}(t) \\
\cdots & \cdots & \cdots \\
h_{\mathrm{mc}_{M_{R^{\prime}}}}(t) & \cdots & h_{\mathrm{mc}_{M_{R}, N_{T}}}(t)
\end{array}\right] *\left[\begin{array}{c}
g_{1}^{(i)}(t) \\
\cdots \\
g_{N_{T}}^{(i)}(t)
\end{array}\right]=0
$$

or equivalently in more compact form:

$$
\mathbf{H}_{\mathrm{mc}}(t) * \mathbf{g}^{(i)}(t)=0
$$

Although given in baseband, (11) ensures SIC even at the RF frontend. Also note that when all elements in a row of $\mathbf{H}_{\mathrm{mc}}(t)$ are corrupted by a common scalar due to receiver phase noise, the solution $\mathbf{g}^{(i)}(t)$ to (11) is not affected.

To find the solution to (10), we need to apply a known notion of vector space in the field of functions of time. The rank $r_{\mathrm{H}(t)}$ of the matrix $\mathbf{H}_{\mathrm{mc}}(t)$ that are convolutely independent. It follows that $r_{\mathrm{H}(t)} \leq \min \left\{M_{R}, N_{T}\right\}=M_{R}$. The dimension of the solution space of (10), which is also called the dimension of the (right) null space of $\mathbf{H}_{\mathrm{mc}}(t)$, is the number of convolutely independent solutions to (10), which is $d_{\text {null }}=N_{T}-r_{\mathbf{H}(t)} \geq N_{s}$. If $d_{\text {null }}=N_{s}$, we call it a typical case (very likely in practice), or otherwise, if $d_{\text {null }}>N_{s}$, we call it atypical case (not very likely in practice). The number $I$ of the data streams in (9) must be no larger than $d_{\text {null }}$.
In general, for $M_{R} \geq 1$ and $N_{s} \geq 1$, the $i$ th in a set of $N_{s}$ convolutely independent solutions to (12) can be written as

$$
\mathbf{g}^{(i)}(t)=\left[\begin{array}{c}
\overline{\mathbf{g}}^{(i)}(t) \\
\mathbf{0}_{i-1,1} \\
g_{0}^{(i)}(t) \\
\mathbf{0}_{I-1,1}
\end{array}\right],
$$

where $\mathbf{0}_{m, 1}$ is the $m \times 1$ zero vector and $\overline{\mathbf{g}}^{(i)}(t)$ and $g_{0}^{(i)}(t)$ are a solution to $\mathbf{A}(t) * \overline{\mathbf{g}}^{(i)}(t)+\mathbf{b}_{i}(t) * g_{0}^{(i)}(t)=0$, where $\mathbf{A}(t)$ is a square matrix equal to $\mathbf{H}_{\mathrm{mc}}(t)$ without its last $N_{s}$ columns and $\mathbf{b}_{i}(t)$ is the $\left(M_{R}+i\right)$ th column of $\mathbf{H}_{\mathrm{mc}}(t)$. Furthermore, we can choose the solution

$$
\overline{\mathbf{g}}^{(i)}(t)=-\operatorname{adj}\{\mathbf{A}(t)\} * \mathbf{b}_{i}(t),
$$

and $g_{0}^{(i)}(t)=\operatorname{det}\{\mathbf{A}(t)\}$. Both the adjoint $\operatorname{adj}\{\mathbf{A}(t)\}$ and the determinant $\operatorname{det}\{\mathbf{A}(t)\}$ can be obtained analytically in the same way as those of a matrix of numbers as shown in [21] except that all multiplications should be substituted by convolutions. It is important to note that expression (13) does not involve any division but only convolutions and sums.

The solutions shown in (12) are valid for arbitrary $\mathbf{H}_{\mathrm{mc}}(t)$ as long as $\operatorname{det}\{\mathbf{A}(t)\} \neq 0$. This condition can be met if $h_{k, k}(t)$ for $k=1, \ldots, M_{R}$ have the largest norms among $h_{l, k}(t)$ for all $l$ and $k$. To ensure that, we can either place the $M_{R}$ auxiliary transmitting antennas close enough to the $M_{R}$ receiving antennas or directly couple the $M_{R}$ auxiliary generators to the $M_{R}$ receivers at the RF frontend.

In this section, the proposed system is designed to formulate the self-interference based on mutual-coupling model. These self-interference signals are caused by multiple antennas. The proposed system for full-duplex single-channel MIMO system is illustrated in Figure 7. As shown in Figure 7, the self-interference based on mutual-coupling $\mathbf{H}_{\mathrm{mc}_{I}}$ can be written as

$$
\mathbf{H}_{\mathrm{mc}_{I}} \mathbf{s}=\mathbf{H}_{\mathrm{LI}} \mathbf{s}+\mathbf{H}_{\mathrm{NI}} \mathbf{C}_{R} \mathbf{s},
$$

where $\mathbf{s} \in C^{s \times 1}$ is the transmitted signal, $\mathbf{H}_{\mathrm{LI}} \in C^{s \times s}$ is a diagonal matrix that represents the self-interference signals, and $\mathbf{H}_{\mathrm{NI}} \in C^{s \times s}$ is a symmetric matrix that represents the mutual-interference signals caused by the other antennas.

Next, the proposed method to suppress the interference signals is performed as shown in Figure 7. The transmitted signals are coupled to matrix $W$ in order to perform the negative self-interference and mutual-interference signals as closely as possible. Inside matrix $W$, the attenuation and phase shifter are employed to adjust the preknown signals for compensating the self-interference signals and mutualinterference signals. The compensation matrix, $\mathbf{W}$, is given by

$$
\mathbf{W} \mathbf{s}=\mathbf{T}_{x} \mathbf{C}_{T} \mathbf{s}+\mathbf{G s},
$$

where $\mathbf{T}_{x} \in C^{s \times s}$ is a symmetric matrix that represents the mutual-interference signals caused by the other antennas, 


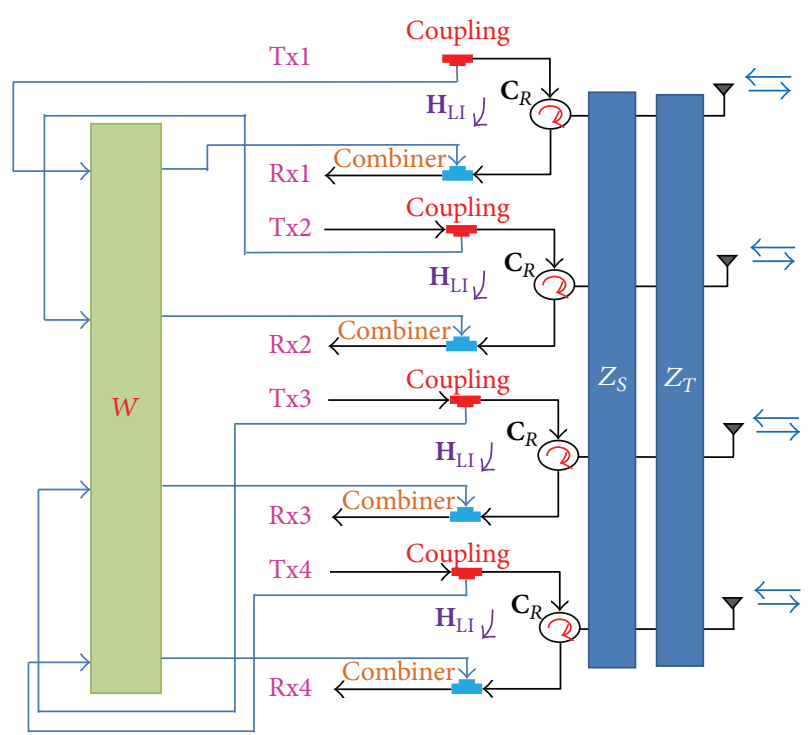

FIgURE 7: Proposed self-interference cancellation for full-duplex single-channel $4 \times 4$ MIMO system based on mutual-coupling model.

$\mathbf{G} \in C^{s \times s}$ is a diagonal matrix that represents the self-interference signals.

Then, the received signal at the destination with the proposed compensation matrix for the interference suppression can be rewritten as

$$
\mathbf{y}=\mathbf{H}_{\mathrm{mc}} \mathbf{x}+\mathbf{H}_{\mathrm{mc}_{I}} \mathbf{s}-\mathbf{W s}+\mathbf{N},
$$

where $\mathbf{N} \sim \mathrm{CN}\left(0, \sigma_{d}^{2} \mathbf{I}_{s}\right)$ is the AWGN contribution at the destination.

\section{Results and Discussion}

5.1. Channel Capacity. In order to investigate the effect of mutual coupling on MIMO capacity, in this section the channel capacity for our $4 \times 4$ MIMO system can be given by (17) [2]. This capacity denotes the average of channel capacity in bps/Hz. Also, we assume the uniform transmitting power for each antenna $\left(E\left\{\mathbf{x x}^{H}\right\}=\left(P_{0} / N_{s}\right) \mathbf{I}_{x}\right)$. Consider

$$
\begin{aligned}
C=\log _{2} \operatorname{det}[ & \mathbf{I}_{s}+\frac{P_{0}}{N_{s}} \mathbf{H}_{\mathrm{mc}} \mathbf{H}_{\mathrm{mc}}^{H} \\
& \left.\times\left(\sigma_{I}^{2} \mathbf{H}_{\mathrm{mc}_{I}}^{\prime} \mathbf{H}_{\mathrm{mc}_{I}}^{\prime H}+\sigma_{d}^{2} \mathbf{I}_{s}\right)^{-1}\right] .
\end{aligned}
$$

We assume that $P_{0}$ is the maximum available power at the source and $N_{s}$ is the power of the self-interference signals.

The performance of channel capacity is presented by considering four cases. The first case is that there is neither self-interference nor mutual-coupling effect (called without interference and MC) in the system. In the second case, there is no interference but including mutual-coupling effect (called without interference and with MC). The third case is the case that the system uses the self-interference cancellation and there is a mutual-coupling effect in the systems (called

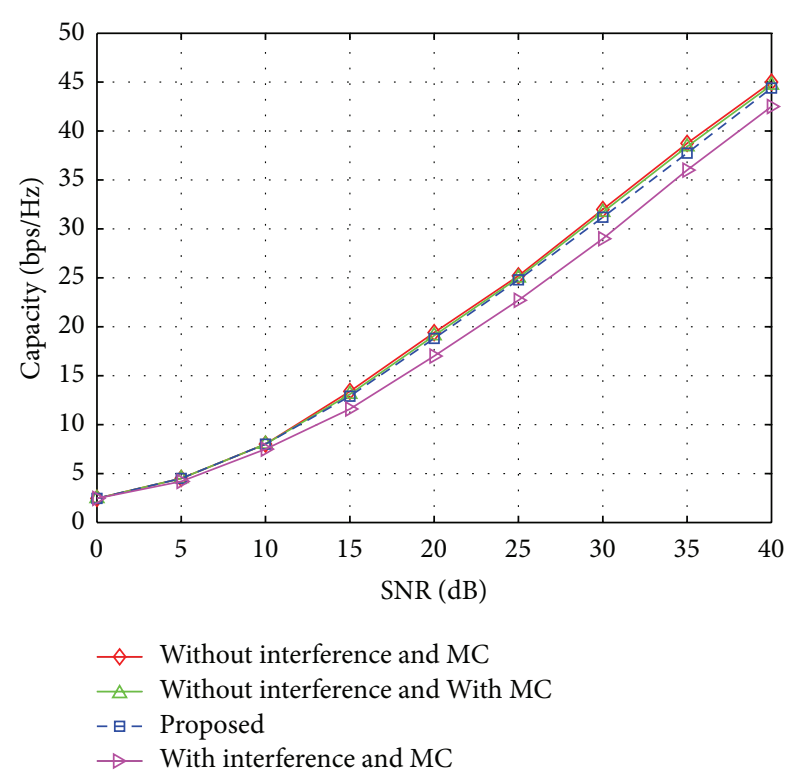

FIGURE 8: Channel capacity versus SNR for $80 \%$ interference reduction.

proposed). For the last case, the system experiences both interference and mutual-coupling effect but no any cancellation technique is applied (called with interference and MC).

The simulation produced by MATLAB programming can be described as follows. The source and the destination are equipped with four transmitting and four receiving antennas; that is, $s=x=4$. We assume that the source-destination channels experience Rayleigh fading. Hence, the new channel matrix $\mathbf{H}_{\mathrm{mc}}$ is an independent matrix containing independent identically distributed (i.i.d.) entries in which the random distribution is explained by $\mathrm{CN}(0,1)$. For the self-interference channels, they also experience Rayleigh fading. Hence, the self-interference channel matrices $\mathbf{H}_{\mathrm{LI}}$ and $\mathbf{H}_{\mathrm{NI}}$ are independent matrices containing independent identically distributed (i.i.d.) entries distributed as $\mathrm{CN}(0,1)$. For simplicity, we assume that the noise variances are equal in each antenna, $\sigma^{2}$.

Figure 8 shows the channel capacity versus SNR for $80 \%$ interference reduction when the MIMO system is affected by mutual coupling. It can be noticed that the proposed technique lies between with and without the interference suppression. The channel capacity of the proposed system is about $0.70 \mathrm{bps} / \mathrm{Hz}$ (at SNR $=20 \mathrm{~dB}$ ) higher than the system with self-interference and the system with mutual coupling. In Figure 9, the relation between capacity and the percentage of interference reduction is presented. It can be noticed that the channel capacity of the proposed system requires only $90 \%$ interference reduction to achieve the capacity close to the system without any interference.

5.2. Self-Interference Reduction. The work in [6, 8, 17, 22-25] shows that a single-channel full-duplex system can be worked by using the method of self-interference cancellation. Two key techniques are RF interference cancellation (RFIC) and 


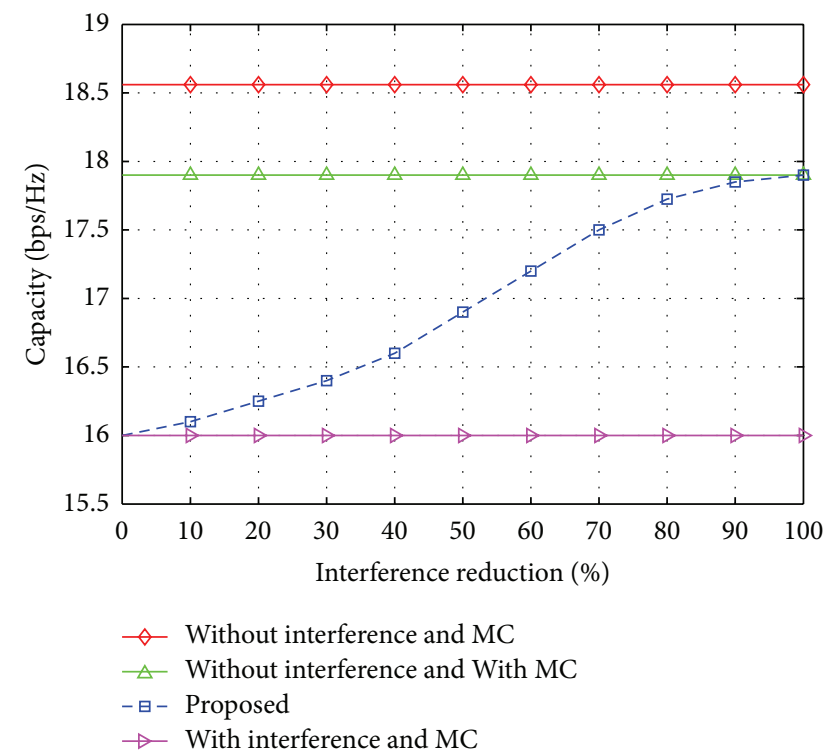

FIGURE 9: Relation between channel capacity and interference reduction for $\mathrm{SNR}=20 \mathrm{~dB}$.

digital interference cancellation (DIC) which utilize the signals from both the transmitting and receiving paths. Figure 10 presents the signal diagram of self-interference cancellation consisting of both RFIC and DIC. RFIC uses the knowledge of transmitting signals to cancel the self-interference in the $\mathrm{RF}$ signal before it is digitized. For analog cancellation, the amplitudes from two paths have to be perfectly matched at the receiver. Then, the phase of the two signals would be ideally differed by the exact $\pi$. To cancel self-interference, the best performing prior design is obtained. The authors gain the inverse of the transmitted signal using phase shifter and attenuator, dynamically adjusting the attenuation and phase of the inverse signal to match the self-interference leaking from circulator. After combining both inverse and leak signals, the received signal can be passed through the processing unit with the minimum effect of self-interference.

In measurement, the operating frequency band is on $2.45 \mathrm{GHz}$ in order to match with a practical wireless channel as IEEE 802.11. The measurement has been performed to investigate the concept of a single-channel full-duplex wireless system. The results show that the system can reduce the self-interference about $-75 \mathrm{~dB}$. This reduction is good enough to investigate the concept of a single-channel fullduplex wireless system. The results show that the system can reduce the self-interference about $-75 \mathrm{~dB}$. This reduction is good enough to transmit and receive on the same frequency at the same time. However, we have proposed the selfinterference suppression for MIMO system in which the self-interference signals are caused by mutual coupling. The proposed suppression technique can also be applied to the MIMO system by separating the multiple antennas into individual measurement. In this paper, the RFIC is performed according to the diagram shown in Figure 10. Then the DIC is performed inside USRP (Universal Software Radio Peripheral) processors.

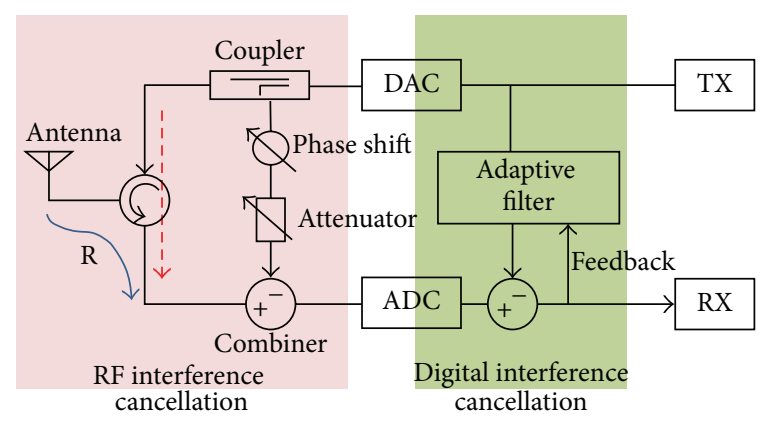

FIGURE 10: Block diagram of the proposed system in practice.

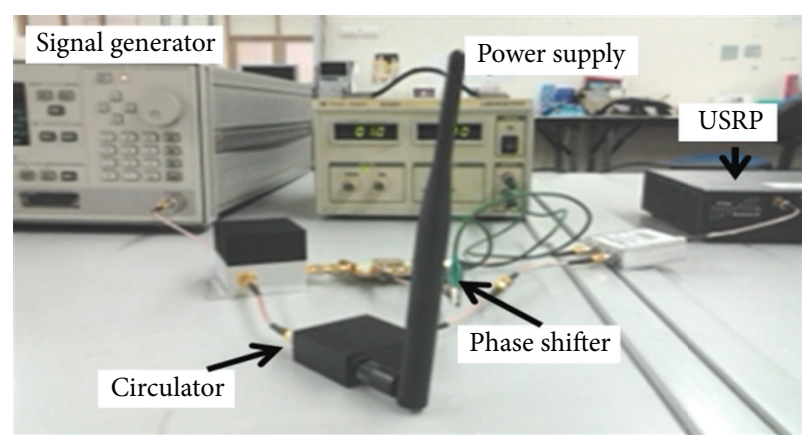

FIGURE 11: Photograph of experimental scenario.

The Universal Software Radio Peripheral (USRP) is a platform developed by Ettus Research LLC. Inside the USRP, there are two main components. The first component is a mother board containing an Altera Cyclone EP1C12 Field Programmable Gate Array (FPGA). It has 4 ADCs with 12 bits per sample and 4 DACs with 14 bits per sample. The second component is a daughter board that all working processes are in a field of RF-Front End. This paper employs XCVR2450 daughter board which responses to radio frequency in dual band, both $2.4 \mathrm{GHz}$ and $5.9 \mathrm{GHz}$. All components are assembled in one USRP box using $3 \mathrm{~A}-6 \mathrm{~V}$ power supply. USRP is connected to the host PC via USB 2.0 (Universal Serial Bus 2.0).

The digital interference cancellation technique in our design employs a finite impulse response (FIR) filter to cancel the remainder of the self-interference signals after RF interference cancellation. The transmitted digital samples are passed through the FIR filter to create digital interference cancellation samples which are subtracted from the received samples to further clean interference from the received signal.

Figure 11 shows the photograph of the experimental scenario for measuring the self-interference signal. The block diagram of each antenna with both RFIC and DIC is shown in Figure 10.

Figure 12 shows the measured spectrum of self-interference signal. In Figure 12(a), the spectrum of the selfinterference leakage without any cancellation is noticeably high. In Figure 12(b), the measured spectrum of selfinterference signal with RF interference cancellation is reduced by $58 \mathrm{~dB}$. In Figure 12(c), the measured spectrum of 


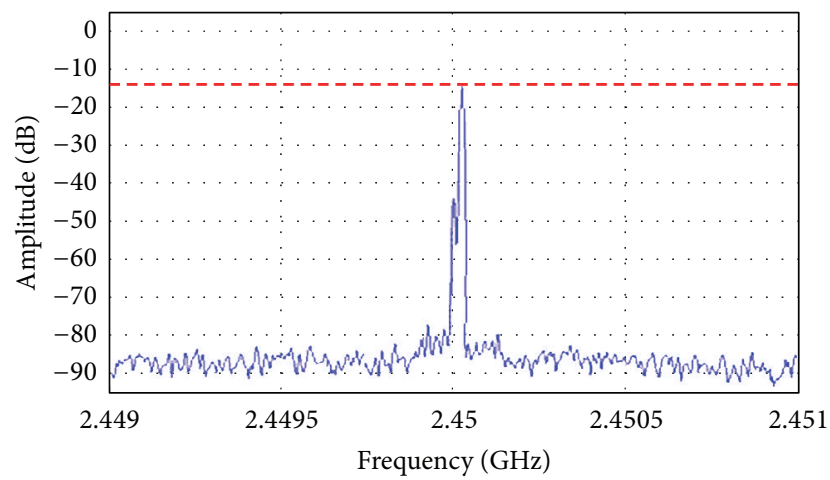

(a)

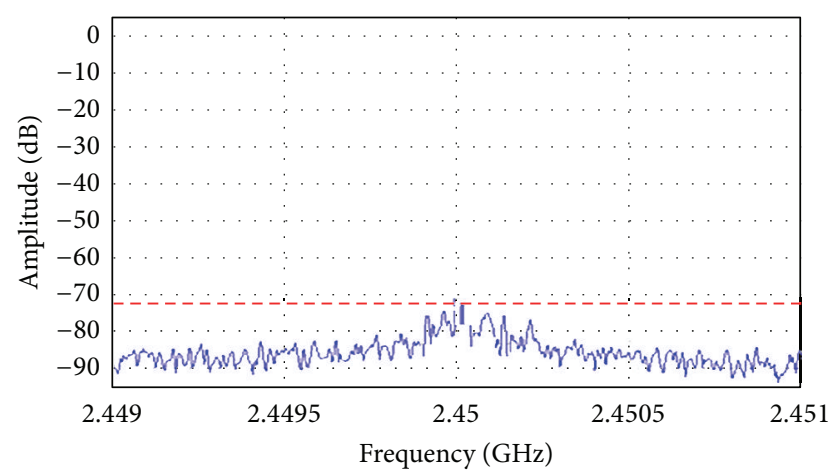

(b)

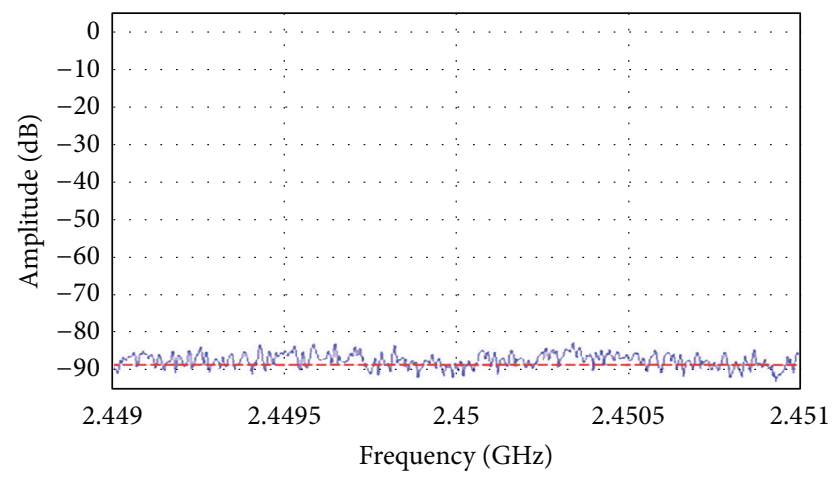

(c)

Figure 12: Measured spectrum of the self-interference signal (a) without any cancellations, (b) with RF interference cancellation but without digital interference cancellation, and (c) with both RF and digital interference cancellations.

self-interference signals with both RF and digital cancellations is very low and close to the noise floor level with the reduction of $75 \mathrm{~dB}$. At this stage, the self-interference signal is low enough to provide a little impact on the desirably received signals. It means that the full-duplex system can be operated on the same channel at the same time because the self-interference is treated to be a noise for both forward and reserve links. Consequently, the throughput can be doubled by using our proposed method.

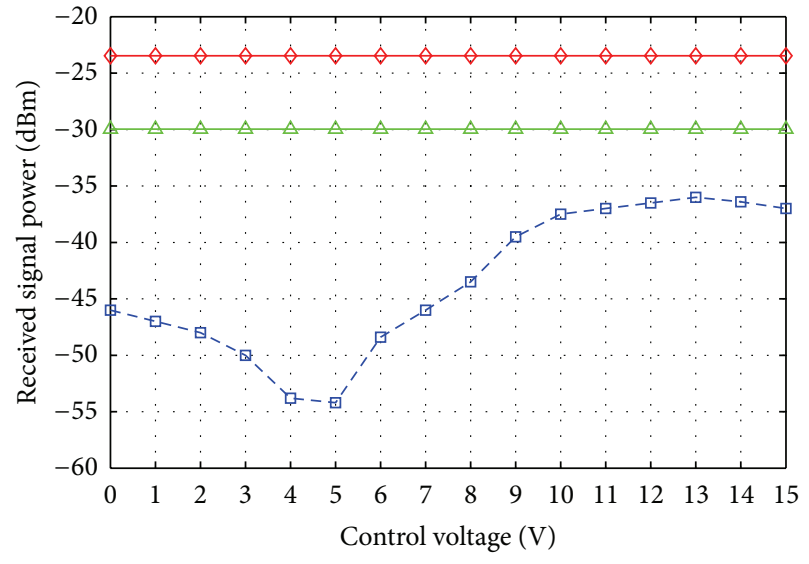

$\triangleleft$ Self-interference signals (TX1 only)
$\triangleleft-$ Self- and mutual-interference signals
$-\square-$ Proposed

FIGURE 13: The received signal power of interference signals at Rxl.

5.3. Mutual-Interference Reduction. In the previous section, the reduction of self-interference power is observed. However, in MIMO system, there are other interference signals called mutual-interference signals. The proposed work also considers the reduction of mutual interference as well. By using the same measurement as previous section but increasing all sets for $4 \times 4$ MIMO operation, the mutual-interference power can be observed. The operating frequency band is on $2.45 \mathrm{GHz}$ for all transmitting antennas. The attenuations and phase shifters are employed to perform the suitable matrix W which is illustrated in Figure 7. The power inputs of Txl, $\mathrm{Tx} 2, \mathrm{Tx} 3$, and $\mathrm{Tx} 4$ are equal. Figure 13 shows the measured powers from Rxl output. There are three curves presented in Figure 13. The first curve is named as self-interference signals because the signal is sent by only Txl while there is no input power for Tx2, Tx3, and Tx4. This is the same situation as in the previous section except that it might be the effect of mutual coupling from the neighbour antenna. For the second curve named as self- and mutual-interference signals, there are equal powers for Txl, Tx2, Tx3, and Tx4, but there is no matrix $\mathbf{W}$ in the system. It can be observed that the total power of this curve is higher than the first curve. In the third curve named as proposed, the matrix $\mathbf{W}$ is performed to suppress both self-interference and mutual-interference signals. In this measurement, there is no signal coming from the other side. Hence, Rxl should not receive any power if matrix $W$ works very well. In our measurement, it can be noticed that the received power of the proposed method is the least. The self-interference and mutual-interference signals can be reduced by adjusting the suitable voltage control for phase shifter. Actually, there are four phase shifters related to this curve and all are needed to be properly adjusted at the same time. To explain the mechanism of phase adjustment, only one voltage control has been presented in Figure 13. It can be clearly seen that the right voltage offers the maximum reduction of interference signals. At control voltage $4-5 \mathrm{~V}$, 


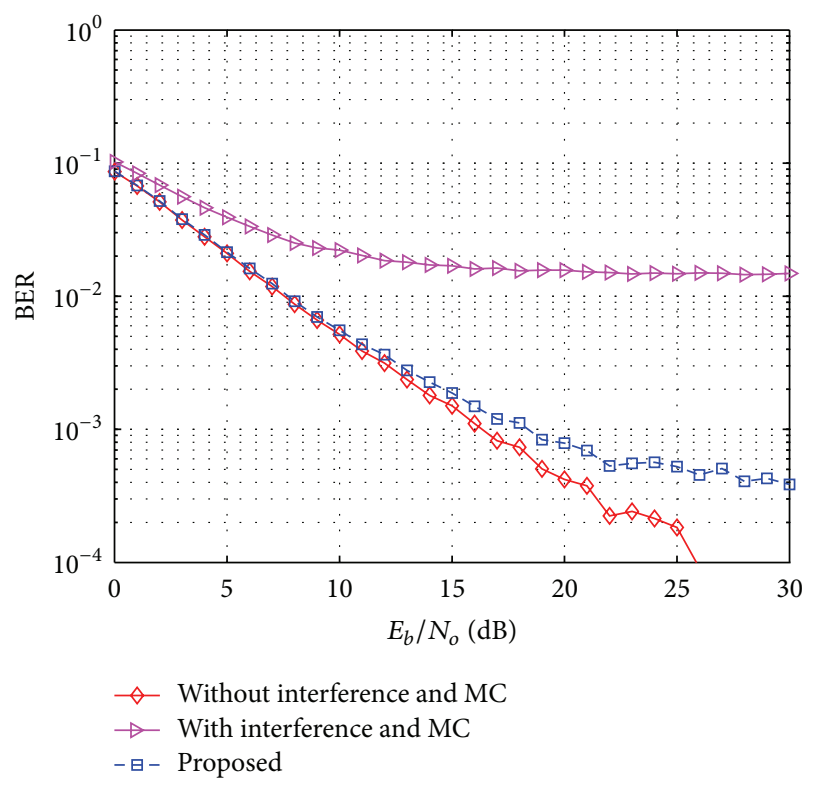

FIGURE 14: BER performance for $4 \times 4$ MIMO system.

the received power of self-interference and mutual-interference signals is reduced by $31 \mathrm{~dB}$.

5.4. Performance of Proposed MIMO System. After getting the suitable matrix $W$, the other side of communication sends the data signal through the wireless $4 \times 4$ channel. It is a fact that the channel capacity is a theoretical quantity which cannot be directly measured. In practice, throughput and bit error rate (BER) are two indicators to judge the merit of system. In this paper, BER can be obtained by using the zero forcing technique to decode the data. All signals are sent with QPSK modulation. Figure 14 shows BER performance for $4 \times$ 4 MIMO system. It is clearly seen that the proposed technique can provide a similar BER to the system without interference when $\mathrm{Eb} / \mathrm{No}$ is less than $15 \mathrm{~dB}$. Even though $\mathrm{Eb} / \mathrm{No}$ is more than $15 \mathrm{~dB}$, the proposed system still significantly improves the BER performance in comparison with the system with interference.

Note that even the BER of proposed system is nearly the same as that of the system without interference but the throughput of proposed system is a double of that of normal full-duplex system. This is because the proposed MIMO system can transmit and receive at the same time and on the same frequency.

\section{Conclusions}

In this paper, we proposed the method of self-interference cancellation for full-duplex single-channel MIMO system based on mutual-coupling model. The performance of proposed technique can suppress the self-interference signals by using the preknown interferences which are affected by mutual coupling between antennas. Simulation results illustrate that the proposed system outperforms the system with interference. This implies the success of using the proposed concept for full-duplex single-channel MIMO system. In addition, the measurement results indicate that the selfinterference and mutual-interference reductions are good enough to successfully transmit and receive on the same frequency at the same time in practice. As a result, the proposed throughput can be actually twice the conventional system.

\section{Conflict of Interests}

The authors declare that there is no conflict of interests regarding the publication of this paper.

\section{Acknowledgment}

The authors got a financial support from Thailand Research Fund through the Royal Golden Jubilee Ph.D. Program (Grant no. PHD/0076/2554).

\section{References}

[1] T. Riihonen, S. Werner, and R. Wichman, "Spatial loop interference suppression in full-duplex MIMO relays," in Proceedings of the 43rd Asilomar Conference on Signals, Systems and Computers (Asilomar '09), pp. 1508-1512, Pacific Grove, Calif, USA, November 2009.

[2] Y. Y. Kang and J. H. Cho, "Capacity of MIMO wireless channel with full-duplex amplify-and-forward relay," in Proceedings of the IEEE 20th Personal, Indoor and Mobile Radio Communications Symposium (PIMRC '09), pp. 117-121, Tokyo, Japan, September 2009.

[3] P. Larsson and M. Prytz, "MIMO on-frequency repeater with self-interference cancellation and mitigation," in Proceedings of the IEEE 69th Vehicular Technology Conference (VTC '09), pp. 1-5, Bercelona, Spain, April 2009.

[4] A. Goldsmith, Wireless Communications, Cambridge University Press, Cambridge, UK, 2005.

[5] B. Radunovic, D. Gunawardena, P. Key et al., "Rethinking indoor wireless mesh design: low power, low frequency, fullduplex," in Proceedings of the 5th Annual IEEE Workshop on Wireless Mesh Networks (WiMesh '10), pp. 25-30, Boston, Mass, USA, June 2010.

[6] J. Choi II, M. Jain, K. Srinivasan, P. Levis, and S. Katti, "Achieving single channel, full duplex wireless communication," in Proceedings of the 16th Annual Conference on Mobile Computing and Networking (MobiCom '10), pp. 1-12, Chicago, Ill, USA, September 2010.

[7] N. Singh, D. Gunawardena, A. Proutiere, B. Radunovic, H. V. Balan, and P. Key, "Efficient and fair MAC for wireless networks with self-interference cancellation," in Proceedings of the International Symposium of on Modeling and Optimization in Mobile, Ad Hoc and Wireless Networks (WiOpt '11), pp. 94101, Princeton, NJ, USA, May 2011.

[8] M. Jain, J. I. Choi, T. Kim et al., "Practical, real-time, full duplex wireless," in Proceedings of the 17th Annual International Conference on Mobile Computing and Networking (MobiCom '11), pp. 301-312, Las Vegas, Nev, USA, September 2011.

[9] M. A. Khojastepour, K. Sundaresan, S. Rangarajan, X. Zhang, and S. Barghi, "The case for antenna cancellation for scalable full-duplex wireless communications," in Proceedings of the 
10th ACM SIGCOMM Workshop on Hot Topics in Networks (HotNets-X '11), article 17, Cambridge, Mass, USA, November 2011.

[10] L. Sun, P. Li, M. R. McKay, and R. D. Murch, "Capacity of MIMO systems with mutual coupling: transmitter optimization with dual power constraints," IEEE Transactions on Signal Processing, vol. 60 , no. 2, pp. 848-861, 2012.

[11] T. Nguyen, W. Meng, and H. Wang, "Channel capacity analysis on cooperative MIMO with antenna spatial correlation and multi-path," in Proceedings of the 6th International ICST Conference on Communications and Networking in China (CHINACOM '11), pp. 181-185, Harbin, China, August 2011.

[12] R. M. Legnain, R. H. M. Hafez, I. D. Marsland, and A. M. Legnain, "A novel spatial modulation using MIMO spatial multiplexing," in Proceedings of the 1st International Conference on Communications Signal Processing and their Applications (ICCSPA '13), pp. 1-4, Sharjah, United Arab Emirates, February 2013.

[13] P. Li, L. Sun, M. R. McKay, and R. D. Murch, "Transmitter optimization for MIMO systems with mutual coupling at high SNR," in Proceedings of the 45th Asilomar Conference on Signal Systems and Computers (ASILOMAR '11), pp. 1058-1588, Pacific Grove, Calif, USA, November 2011.

[14] H.-B. Shi, S. Gong, and T.-C. Zheng, "The effect of mutual coupling on the channel performance of MIMO communication system," in Proceedings of the 10th International Symposium on Antennas Propagation \& EM Theory (ISAPE '12), pp. 335-339, Xian, China, October 2012.

[15] S. Lu, H. T. Hui, and M. Bialkowski, "Optimizing MIMO channel capacities under the influence of antenna mutual coupling," IEEE Antennas and Wireless Propagation Letters, vol. 7, pp. 287-290, 2008.

[16] P. Uthansakul, Adaptive MIMO Systems: Explorations for Indoor Wireless Communications, VDM, Berlin, Germany, 2009.

[17] N. Phungamngern, P. Uthansakul, and M. Uthansakul, "Digital and RF interference cancellation for single-channel full-duplex transceiver using a single antenna," in Proceedings of the 10th International Conference on Electrical Engineering/Electronics, Computer, Telecommunications and Information Technology (ECTI-CON '13), pp. 1-5, Krabi, Thailand, May 2013.

[18] E. Telatar, "Capacity of multi-antenna Gaussian channels," European Transactions on Telecommunications, vol. 10, no. 6, pp. 585-595, 1999.

[19] C. A. Balanis, Antena Theory, John Wiley \& Sons, Hoboken, NJ, USA, 3rd edition, 2005.

[20] S. Durrani and M. E. Bialkowski, "Effect of mutual coupling on the interference rejection capabilities of linear and circular arrays in CDMA systems," IEEE Transactions on Antennas and Propagation, vol. 52, no. 4, pp. 1130-1134, 2004.

[21] H. Lutkepohl, Handbook of Matrices, John Wiley \& Sons, New York, NY, USA, 1996.

[22] M. Duarte and A. Sabharwal, "Full-duplex wireless communications using off-the-shelf radios: feasibility and first results," in Proceedings of the 44th Asilomar Conference on Signals, Systems and Computers (ASILOMAR '10), pp. 1558-1562, Pacific Grove, Calif, USA, November 2010.

[23] M. A. Khojastepour and S. Rangarajan, "Wideband digital cancellation for full-duplex communications," in Proceedings of the 46th Asilomar Conference on Signals, Systems and Computers (ASILOMAR '12), pp. 1300-1304, Pacific Grove, Calif, USA, November 2012.
[24] M. Duarte, C. Dick, and A. Sabharwal, "Experiment-driven characterization of full-duplex wireless systems," IEEE Transactions on Wireless Communications, vol. 11, no. 12, pp. 4296-4307, 2012.

[25] N. Li, W. Zhu, and H. Han, "Digital interference cancellation in single channel, full duplex wireless communication," in Proceedings of the 8th International Conference on Wireless Communications, Networking and Mobile Computing (WiCOM '12), pp. 1-4, Shanghai, China, September 2012. 

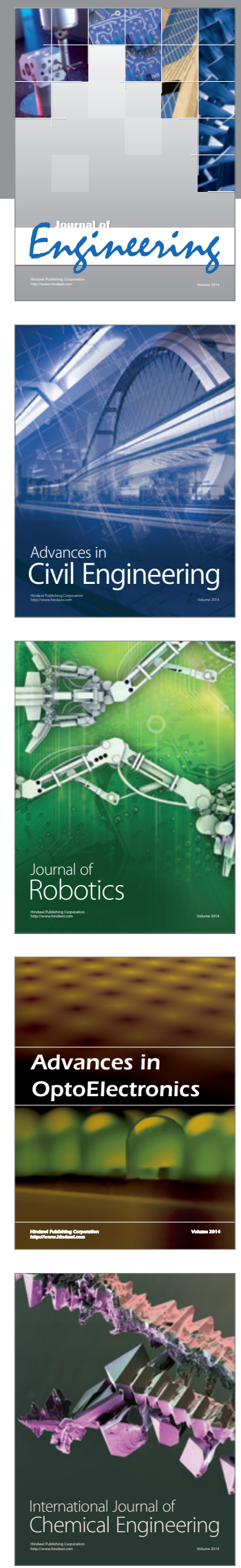

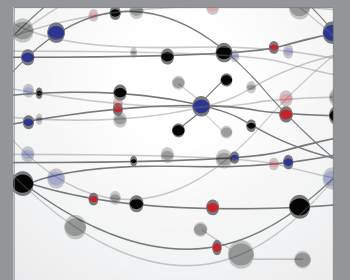

The Scientific World Journal
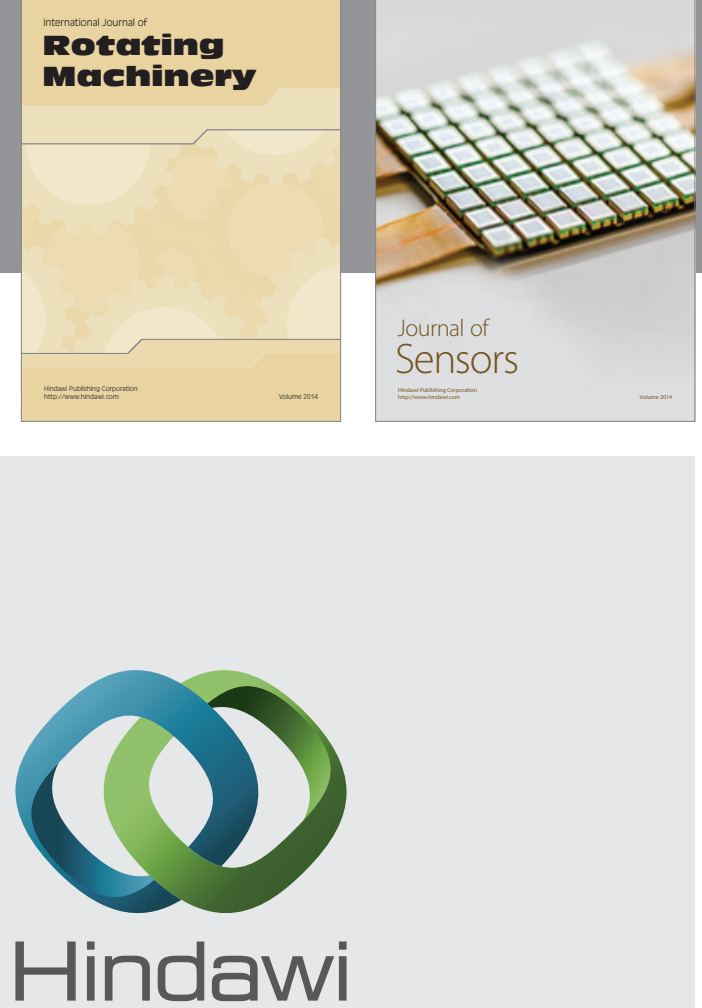

Submit your manuscripts at http://www.hindawi.com
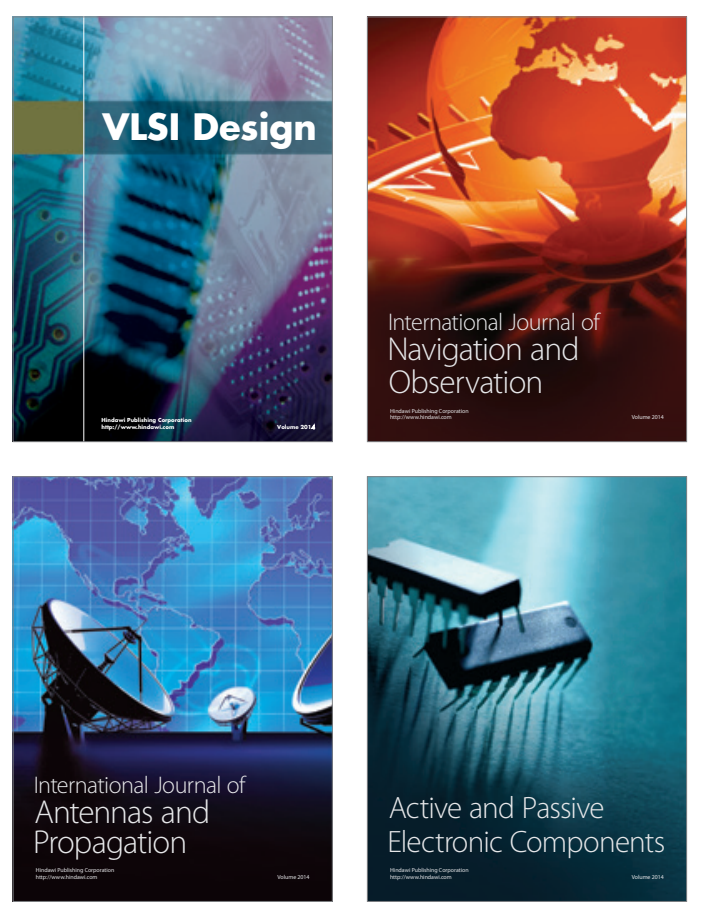
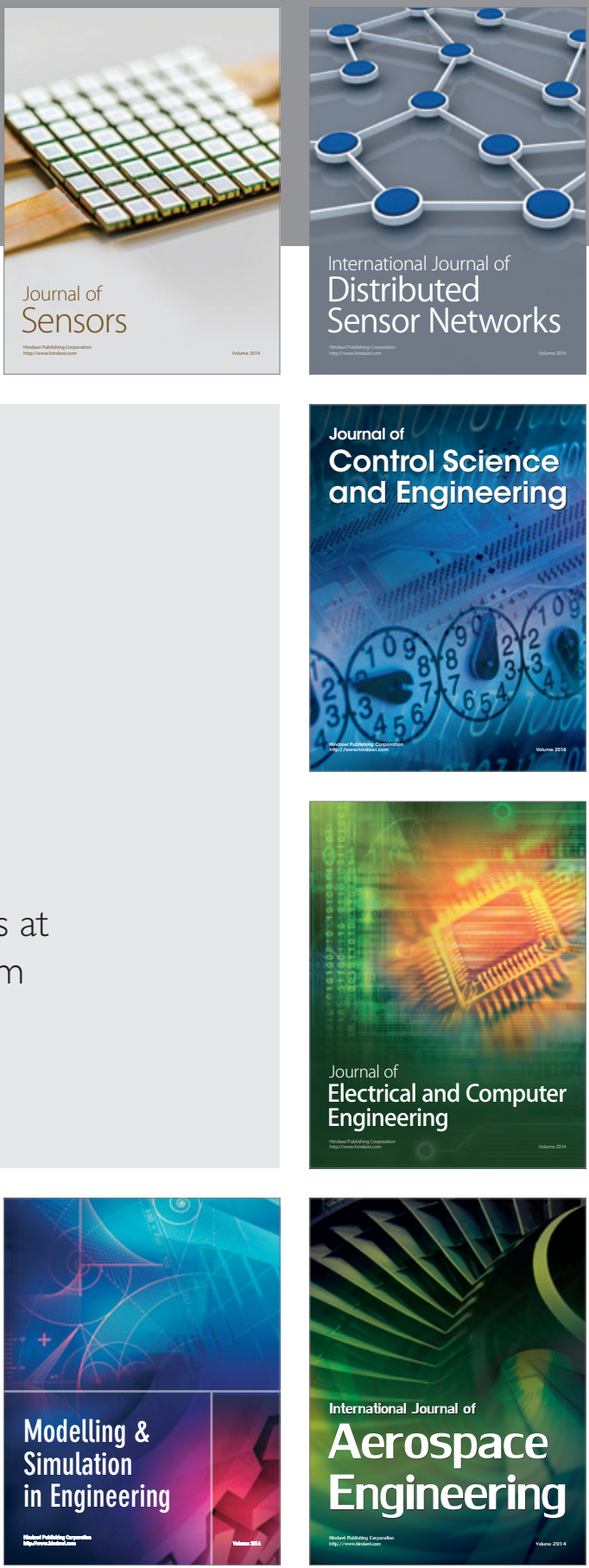

Journal of

Control Science

and Engineering
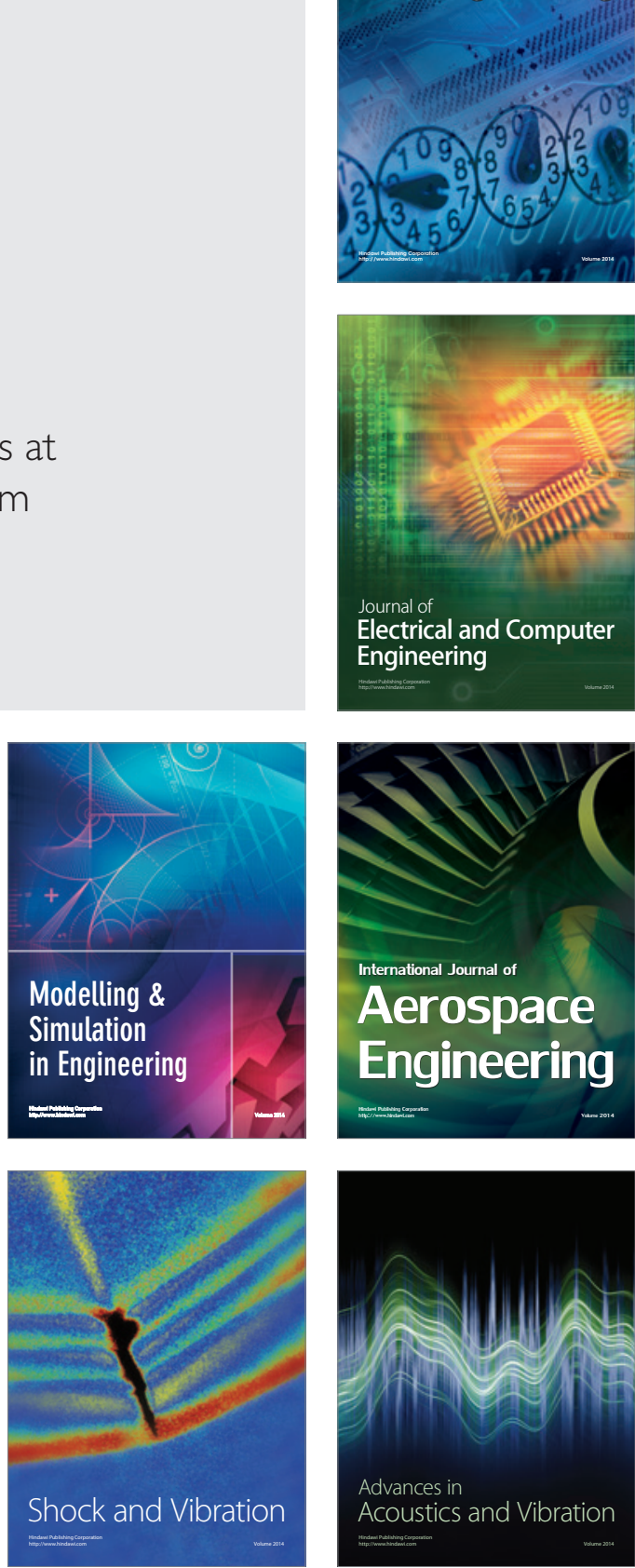